\title{
Implante percutáneo de válvula aórtica sobre válvula protésica estenótica valve in valve con técnica de cracking: primer reporte de un caso en Argentina
}

\author{
Percutaneous implantation of prosthetic aortic valve using cracking \\ technique: first report in Argentina
}

Carlos Fernández-Pereira', Juan Mieres, Hernán Pavlovsky¹, Laura Bidegain², Alfredo E. Rodríguez¹

\section{RESUMEN}

El implante de válvula aórtica percutáneo en pacientes con previa válvula protésica (valve in valve - $V$ in $V$ ) ha sido frecuentemente reportado. En casos de válvula protésica de diámetros pequeños se ha postulado el uso de la técnica de cracking para obtener una mejor área efectiva valvular post-TAVI.

Se presenta el caso de $V$ in $V$ con previa fragmentación de la válvula protésica, cracking, con balón no complaciente en una paciente que había sido operada con cirugía convencional de reemplazo valvular 6 años antes.

La técnica de cracking fue efectiva, inmediatamente después se implantó una válvula protésica percutánea con diámetros mayores, $23 \mathrm{~mm}$, que el anillo original de la válvula protésica, lográndose una efectiva área aórtica posimplante. La paciente tuvo alta domiciliaria 48 horas después.

A nuestro conocimiento, este es el primer caso en la Argentina en que se utilizó esta técnica.

Palabras claves: TAVI, valve in valve, técnica de cracking, estenosis valvular aórtica, cirugía aórtica valvular.

\begin{abstract}
Aortic valve implantation percutaneously (TAVI) in patients with previous prosthetic valve ( $\mathrm{V}$ in $\mathrm{V}$ ) has been frequently reported. In cases of small prosthetic valve diameters has been postulated the use of "cracking" technique to obtain a best effective valve post TAVI. The case of $\mathrm{V}$ in $\mathrm{V}$ with prior fragmentation of the prosthetic, "cracking", valve non compliant balloon in a patient who had been operated with conventional surgery of valve replacement 6 years ago. "Cracking" technique was effective and immediately after a percutaneous implantation of a valve with larger diameter than the prosthetic ring, $23 \mathrm{~mm}$, achieving an effective aortic area post implant. The patient was discharged after 48 hours. To our knowledge this is the first case in Argentina with this technique.
\end{abstract}

Key words: TAVI, valve in valve, cracking technique, aortic valve stenosis, surgical aortic valve replacement.

Revista Argentina de Cardioangiología Intervencionista 2019;10(2):78-82. https://doi.org/10.30567/RACI/201902/0078-0082

\section{INTRODUCCIÓN}

El implante de válvula aórtica percutáneo se ha transformado probablemente en el mayor adelanto tecnológico de los últimos 15 años en cardiología intervencionista, con una importante y rápida evolución tanto tecnológica como de sus indicaciones clínicas ${ }^{1,2}$.

Recientemente hemos visto reportados estudios aleatorizados en pacientes de riesgo intermedio y bajo con resultados similares y aún más favorables que la cirugía convencional de reemplazo valvular aórtico (SAVR: surgical aortic valve replacement $)^{3-5}$.

Los pacientes que presentan estenosis protésica aórtica por una SAVR previa conforman un grupo que también se ha convertido en indicación de esta técnica percutánea de valve in valve $(V$ in $V)$ con resultados muy satisfactorios ${ }^{6,7}$, aunque no es una indicación establecida en las guías de tratamiento. El anillo valvular protésico producto de la cirugía de reemplazo previa es un potencial problema para el correc-

\footnotetext{
1. Departamento de Cardiología Intervencionista Sanatorio Otamendi/Las Lomas/Clínica IMA

2. Técnica de Tecnology SA

$\triangle$ Correspondencia:Dr. Carlos Fernández-Pereira.cfernandezpereira@centroceci. comar
}

Conflicto de intereses: La técnica Laura Bidegain es empleada de Tecnology SA, firma representante en Argentina de Edwards Lifesciences. Los otros autores no presentan conflictos de intereses.

Recibido: 15/04/2019|Aceptado: 06/05/2019 to implante percutáneo de la nueva válvula tanto en los casos con insuficiencia valvular por leak como en la estenosis de la válvula.

El propósito de esta comunicación es presentar un implante valvular percutáneo en un paciente previamente sometido a cirugía valvular utilizando la técnica de cracking, que a nuestro conocimiento es el primer caso realizado y reportado en nuestro país. Este paciente corresponde al 20\% de los implantes $V$ in $V$ de nuestro grupo (1/5 pacientes) sobre un total de 114 procedimientos de TAVI realizados desde el año 2009.

\section{CASO CLÍNICO}

Se trata de una paciente de 79 años con antecedentes de SAVR en el año 2013. En ese momento se le implantó durante el procedimiento de SAVR una prótesis Biocor de Saint Jude No 21 (Abbott/Saint Jude, USA). Esta es una prótesis de tejido biológico-porcino que va montada y fijada sobre un stent. Su durabilidad ha sido demostrada en seguimientos hasta 20 años, aunque en algunos casos se vio, dentro de los 7 años, deterioro y mal función de la válvula ${ }^{8-9}$.

La paciente hasta un año atrás permaneció asintomática haciendo su actividad habitual. Desde entonces comienza con disnea a los esfuerzos que se hace progresiva y actualmente agrega angina de esfuerzo.

El ecocardiograma mostró una severa estenosis protésica valvular aórtica con insuficiencia valvular leve y con calcificación de la prótesis, así como severa calcificación mitral. 


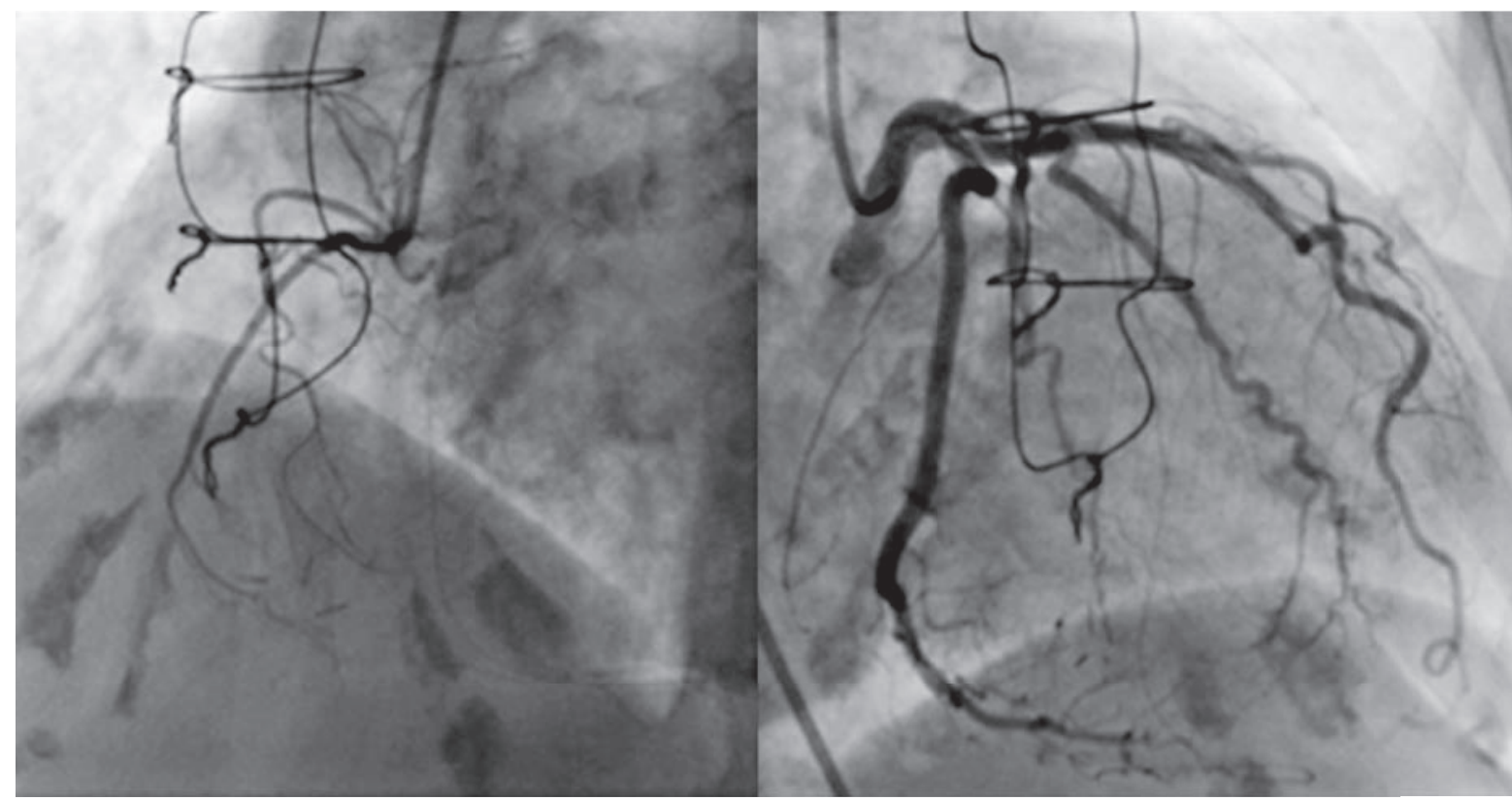

Figura 1. Cinecoronariografía que muestra arterias coronarias sin lesiones significativas.

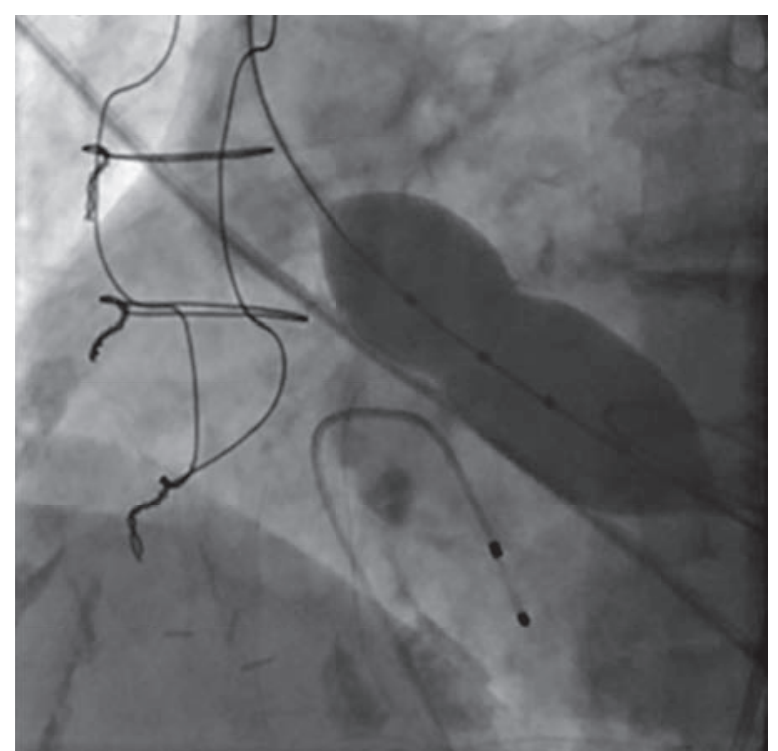

Figura 2. Balón "no complaciente" con la indentación superior marcando la resistencia del stent perivalvular de la prótesis (flecha).

La velocidad pico aórtica fue de $4,36 \mathrm{~m} / \mathrm{s}$, gradiente máximo de $76 \mathrm{mmHg}$, medio de $40 \mathrm{~m} / \mathrm{s}$ y un área de $0,75 \mathrm{~cm}^{2}$. Se le realiza angiotomografía contrastada de cavidades cardíacas y coronarias que evidencia severa calcificación de arteria descendente anterior (DA) con probable estenosis significativa en la arteria DA y una válvula protésica en posición aórtica con estenosis significativa (anilloaórtico de $19 \times 19 \mathrm{~mm}$ ) y con imagen de pannus y trombos de diversos estadios.

En este momento nos es referido el paciente al sanatorio Otamendi para su diagnóstico definitivo y tratamiento.

La paciente es primariamente seleccionada para cinecoronariografía y eventual angioplastia a la arteria DA, dado el antecedente de su angina de esfuerzo y la imagen sugestiva en la angiotomografía.

La cinecoronariografía no evidenció lesiones coronarias significativas en los vasos coronarios (Figura 1).

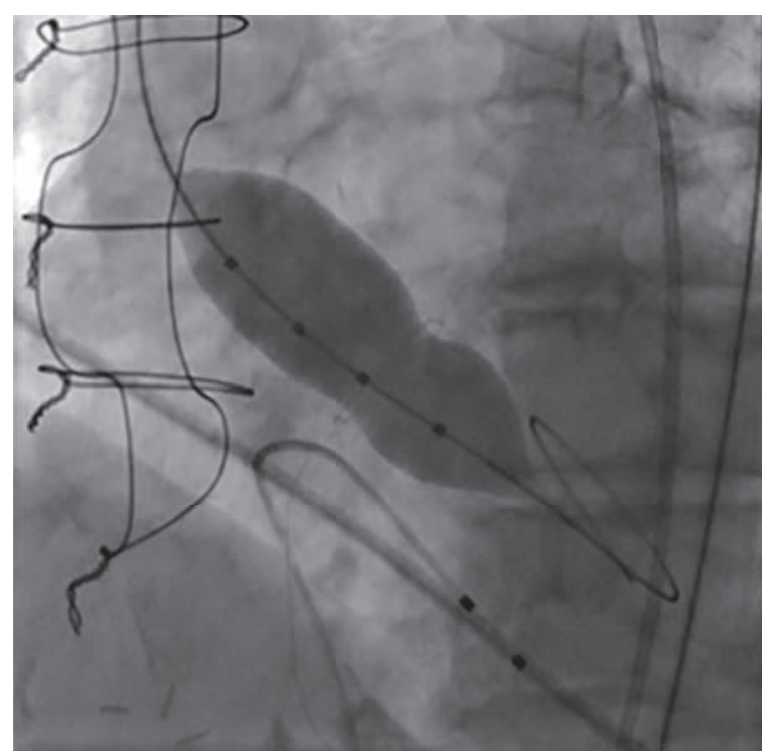

Figura 3. Balón "no complaciente" con la indentación inferior marcando la resistencia del stent perivalvular de la prótesis (flecha).

Con el diagnóstico de estenosis protésica aórtica severa y dado el alto riesgo clínico de acuerdo a la evaluación del heart team para cirugía convencional - presentaba EuroSCORE logístico de $32 \%$, STS de $11 \%$ y test de fragilidad con diagnóstico de "no frágil"10 - a la paciente se le indica implantación percutánea de válvula aórtica.

En este punto la indicación era entre válvula aórtica autoexpandible de $23 \mathrm{~cm}$ o válvula balón expandible de 20; en ambos casos la probabilidad de tener como resultado una estenosis valvular residual importante era alta dada la presencia de un anillo con stent previo de sostén presente en la prótesis quirúrgica de $21 \mathrm{~mm}^{8}$ lo que anticipaba un área valvular efectiva muy estrecha.

Por este motivo se decide la realización de la técnica de fragmentación -cracking- del stent de la válvula previamente implantada como paso previo al TAVI ${ }^{11}$.

Para esta finalidad se usó un balón "no complaciente" de 22 mm Mullins X (NUMED, Canadá) el cual una vez posicio- 


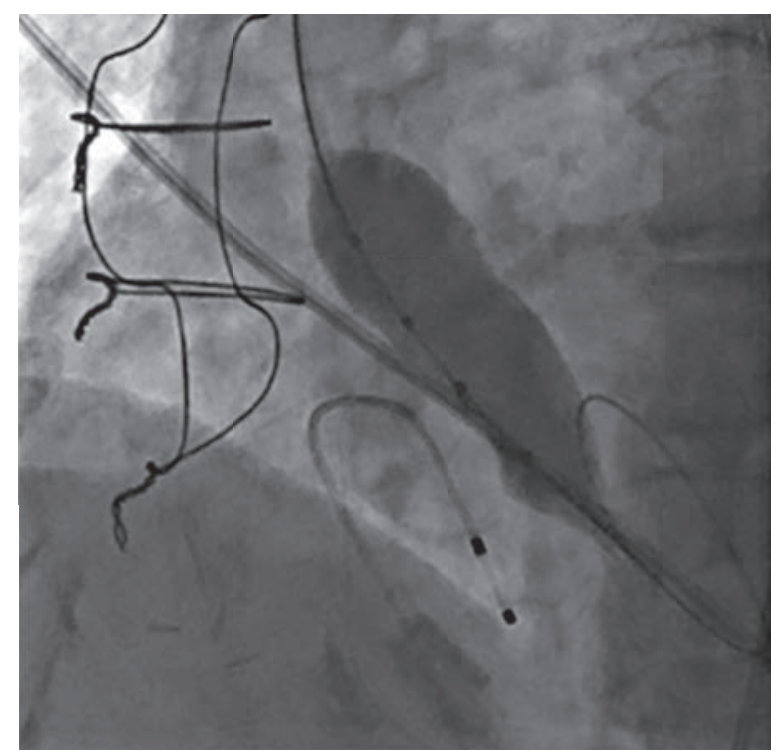

Figura 4. Balón "no complaciente" en el momento del cracking sin indentación (flecha).

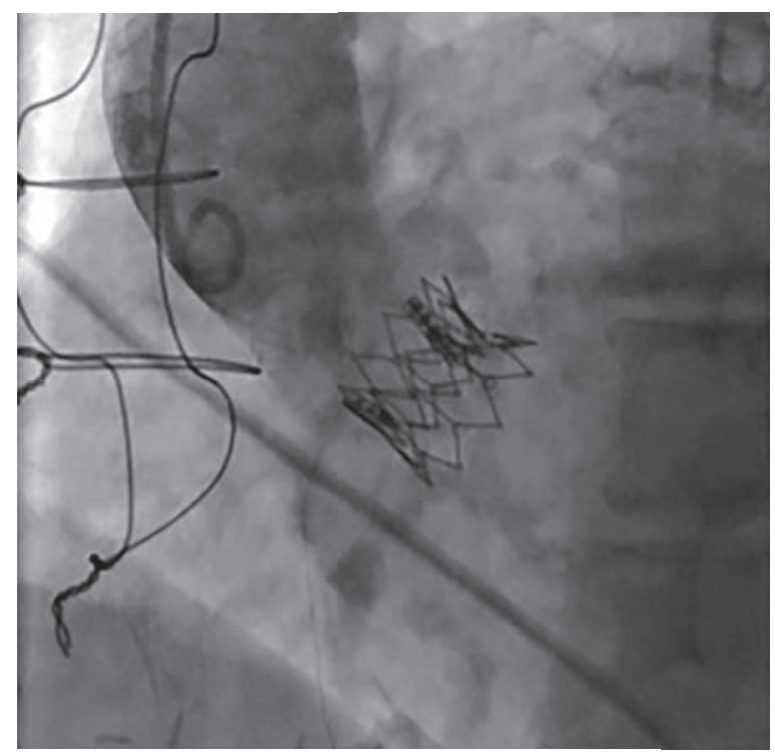

Figura 6. Aortograma posimplante donde se observa la correcta implantación de la válvula sin reflujo perivalvular.

nado sobre la válvula protésica se insufló bajo marcapaseo continuo a 8 ATM en 7 oportunidades sin lograr la apertura del anillo, como se observa en la indentación del balón (Figuras 2 y 3). El cracking de la válvula protésica (Figura 4) se produjo en el octavo intento a una presión de 10 ATM; esto se visualizó mediante fluoroscopia y además por la disminución de la presión en forma súbita en el insuflador.

El inflado del balón que permitió el cracking a diferencia de las anteriores insuflaciones, se hizo en forma lenta a fin de evitar el deslizamiento del mismo que ocurrió en las insuflaciones previas y que impedía ejercer la mayor presión con la porción media central del balón (Figuras 2 y 3 ).

Inmediatamente al cracking se procede a la implantación de una válvula balón-expandible Sapien XT23 (Edwards Lifesciences Corp, USA). El tiempo total transcurrido entre el cracking y la implantación de la válvula fue de 4 minutos 31 segundos.

Luego de la implantación el paciente queda casi sin gradiente, como se pudo ver en la toma de presiones simultáneas posimplante y sin insuficiencia valvular por angiografía (Figu-

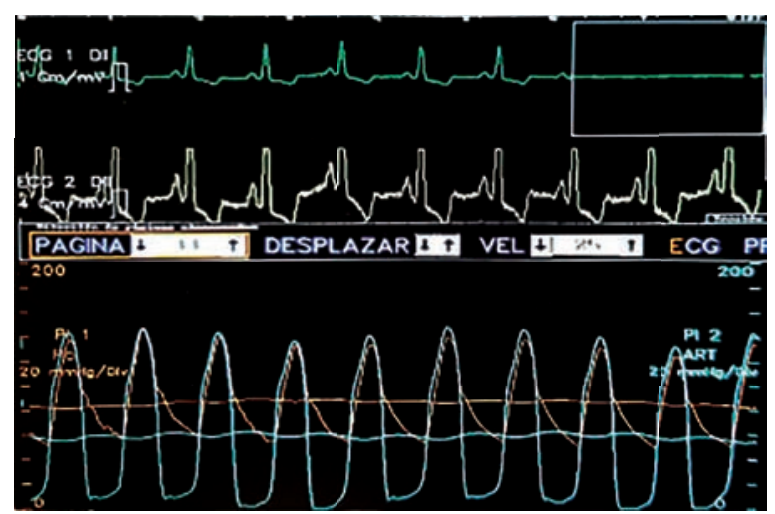

Figura 5. Presiones simultáneas aórtica y del ventrículo izquierdo sin prácticamente gradiente luego del implante valvular de la Sapien XT 23.

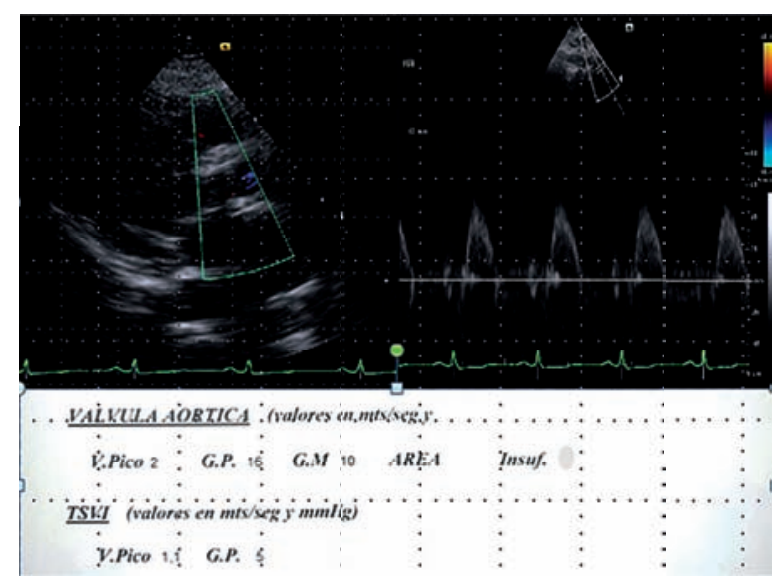

Figura 7. Ecocardiograma 24 hs. posimplante con mínimo gradiente, velocidad de $2.0 \mathrm{~m} / \mathrm{s}$ y un gradiente medio de $10 \mathrm{mmHg}$.

ras 5 y 6). La ecocardiografía de control mostró buena implantación valvular, con gradiente máximo de $16 \mathrm{mmHg}$ y medio de $10 \mathrm{mmHg}$ y una velocidad de $2,0 \mathrm{~m} / \mathrm{s}$ con una insuficiencia aórtica leve (Figura 7).

Al no presentar trastornos de conducción en el ECG (Figura 8), el marcapaso transitorio es retirado inmediatamente posimplante y los introductores arteriales bilaterales y el venoso son retirados y cerrados los accesos mediante compresión manual. No se utilizó disección quirúrgica ni cierre percutáneo de los accesos arteriales y durante el procedimiento la paciente estuvo lúcida y leve sedación.

La paciente fue dada de alta a su domicilio 48 hs posterior al procedimiento sin presentar complicaciones intrahospitalarias.

\section{DISCUSIÓN}

La implantación $V$ in $V$ durante el TAVI es un procedimiento cada vez más utilizado para las válvulas estenóticas o insuficientes previamente implantadas ya sea por SAVR o TAVI. De hecho, como reportamos en la introducción, esto representó en nuestra experiencia el 4,3\% del total de implantes aórticos efectuados. Sin embargo, cuando las válvulas aórticas protésicas son pequeñas y como en este caso se asocian a un anillo metálico inexpandible dado por el hecho de que el tejido biológico está suturado a un stent metálico.La expansión completa de la nueva válvula, si esta es implantada percutáneamente, es muy difícil que se logre; de ahí que en muchos casos quedan con gradientes residuales altos ${ }^{11}$.

En la literatura, se han reportado casos aislados de técnica de cracking durante implantes percutáneos de válvulas pulmonar, 


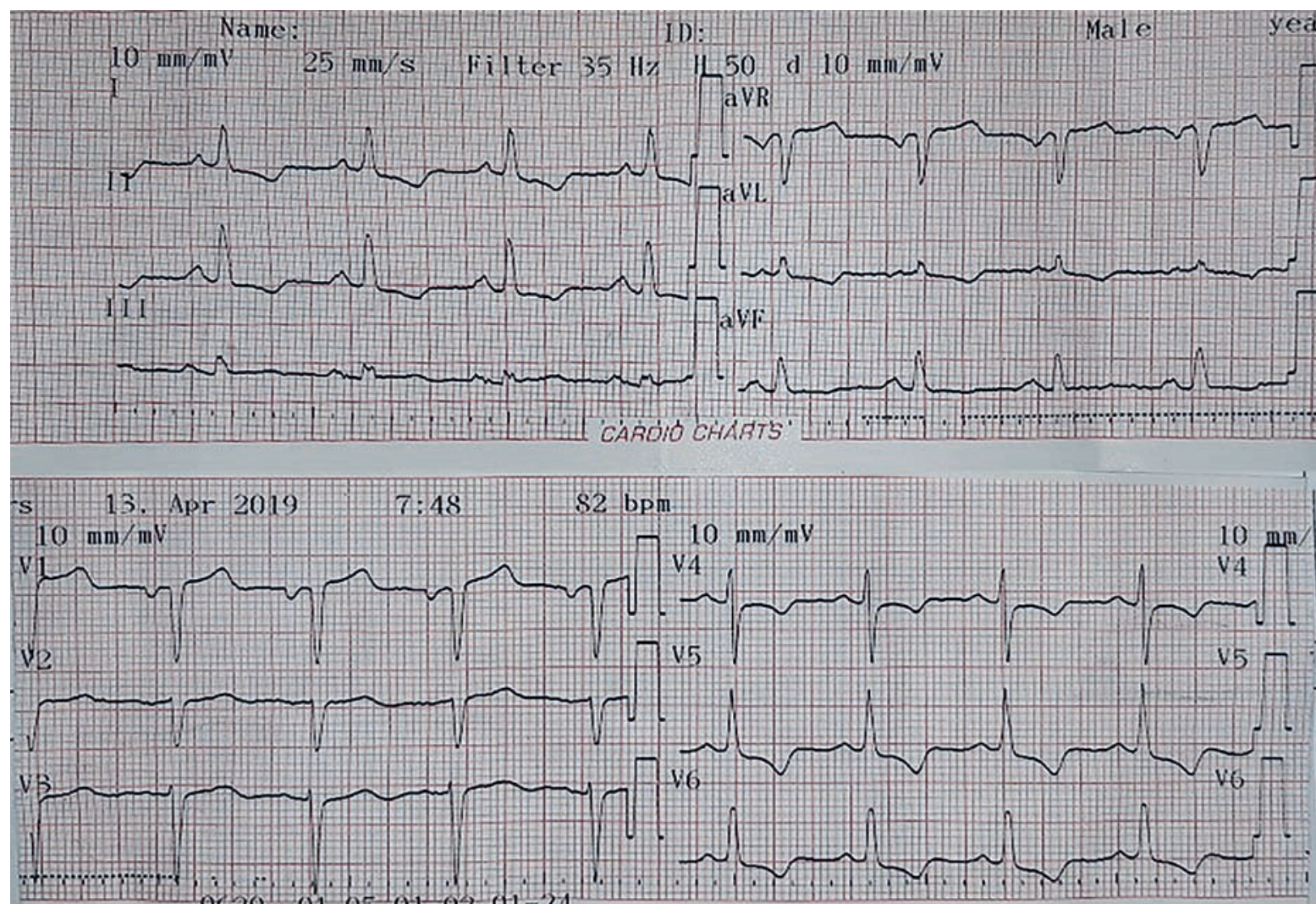

Figura 8. Electrocardiograma posimplante.

tricúspide y aórtica ${ }^{11-13}$ con la finalidad de incrementar el diámetro interno del anillo valvular y así lograr un área efectiva del orificio valvular más grande en la nueva válvula implantada.

La primera descripción fue realizada por Tanase et al. ${ }^{11}$ en el 2014 en 3 casos de estenosis de válvula pulmonar protésica, en los que se utilizaron balones de $20 \mathrm{~mm}$ de diámetro y se pudo lograr un área valvular efectiva de $22 \mathrm{~mm}$.

Posteriormente, Loyalka reportó en Texas, USA, un caso de cracking previo al TAVI en una insuficiencia valvular aórtica periprotésica ${ }^{13}$.

Nuestro caso presentaba una severa estenosis protésica con área de $0,7 \mathrm{~cm}^{2}$ y diámetro interno del anillo medido por tomografía de solo $19 \mathrm{~mm}$, teniendo en cuenta que por las característica de la válvula protésica de estar montada sobre un stent de $21 \mathrm{~mm}$, el área efectiva que se podía obtener iba a ser reducida y probablemente generando importantes gradientes ${ }^{8}$.

Por esta razón es que se decidió usar la técnica de cracking previo al implante. Por este motivo se usó un balón "no complaciente" de $22 \mathrm{~mm}$. La selección de este tipo de balón es muy importante, ya que una ruptura del balón sobre la aorta puede conllevar riesgo de disección de la pared aórtica; de ahí que balones "complacientes' no deberían ser usados para este procedimiento.
Una vez logrado el cracking de la válvula protésica, es muy importante la rápida implantación de la nueva válvula ya que la maniobra sobre la válvula protésica puede causar una insuficiencia valvular severa.

En nuestro caso se pudo lograr implantando rápidamente la nueva válvula sin complicaciones.

Sin embargo, por el motivo recién expuesto, es probable que sólo recomendaremos esta maniobra en casos de válvulas protésicas pequeñas y fuera de la curva de aprendizaje de la técnica de TAVI ${ }^{14,15}$

En conclusión, en presencia de una válvula protésica estenótica de tamaño pequeño como la aquí reportada, el uso de la técnica de cracking con balones "no complacientes" nos permitió la fragmentación del stent perivalvular, la implantación percutánea de una válvula con diámetro mayor y lograr un área aórtica efectiva adecuada como lo demostraron las mediciones angiográficas y ecocardiográficas pos implante.

\section{AGRADECIMIENTOS}

Los autores agradecen al Dr. Adolfo Ferrero por sus consejos con la técnica del cracking.

\section{BIBLIOGRAFÍA}

1. Smith CR, Leon MB, MackMJ, etal. Transcatheterversussurgical aortic-valve replacement in high-risk patients. N Engl J Med 2011;364:2187-98.

2. Adams DH, Popma JJ, Reardon MJ, et al. Transcatheter aortic-valve replacement with a self-expanding prosthesis. N Engl J Med 2014;370:1790-8.

3. Popma J, Deeb M, Yakubov S, et al. Transcatheter Aortic-Valve Replacement witha Self-Expanding Valve in Low-Risk Patients. N Engl J Med 2019;March 17, DOl: 10.1056/NEJMoa1816885.
4. Waksman R, Corsob P, Torgusona R, et al. Transcatheter Aortic Valve Replacement in Low-Risk Patients: One-Year Results from the LRT Trial JACC Cardiovasc Interv 2019 Mar 8. pii: S1936-8798(19)30656-9.

5. MackMJ, Leon MB, Thourani VH, et al PARTNER 3 InvestigatorsTranscatheter Aortic-Valve Replacement with a Balloon-Expandable Valve in Low Risk Patients.N Engl J Med. 2019 Mar 17. doi: 10.1056/NEJMoa1814052. [Epub ahead of print] 
6. Raval J, Nagaraja V, Eslick G, Denniss AR. Transcatheter valve-in-valve implantation: a systematic review of literature. Heart Lung Circ 2014;23:1020-8.

7. Dvir D, Barbanti M, Tan J, Webb JG. Transcatheter aortic valve-in-valve implantation for patients with degenerative surgical bioprosthetic valves. Curr Probl Cardiol 2014;39:7-27.

8. Eichinger W, Hettich I, Ruzicka D, et al. 20-Year Experience with the St. Jude Medical Biocor Bioprosthesis in the Aortic Position. Ann Thorac Surg 2008;86(4):1204-10.

9. Myken P.ATwenty-Year experience of 1712 Patients with the BiocorPorcine Bioprosthesis. J Thorac and Cardiovasc Surg 2009;137:76-81.

10. Green P, Woglom A, Genereux P, et al. The Impact of Frailty Status on Survival After Transcatheter Aortic Valve Replacementin Older Adults With SevereAortic Stenosis. A Single-Center Experience. J Am Coll Cardiol Intv 2012;5:974-81.

11. Tanase D, Grohmann J, Schubert S, et al. Cracking the ring of Edwards Peri- mount bioprosthesis with ultra high pressure balloons prior to transcatheter valve-in-valve implantation. Int J Cardiol 2014;176:1048-9.

12. Loyalka P, Kelsey B, Montgomery BS, et al. Valve-in-Valve Transcatheter Aortic Valve Implantation: A Novel Approach to Treat Paravalvular Leak Ann Thorac Surg 2017;104:e325-7.

13. Brown S, Cools B, Gweilling M. Cracking a tricuspid Perimount bioprosthesis to optimize a second transcatheter Sapien valve-in-valve placement. Catheter Cardiovasc Interv 2016;88:456-9.

14. Mieres J, Menéndez M, Fernández-Pereira C, Rubio M, RodríguezAE. Transapical Implantation of a 2nd-Generation JenaValve Device in Patient with Extremely High Surgical Risk. Case Rep Cardiol 2015;2015:458151.

15. Pavlovsky H, Fernández-PereiraC, Juan Mieres J, et al. Implante percutáneo de válvula aórtica en nonagenarios. Resultados hospitalarios y en el foIlow up. Revista Argentina de Cardioangiología 2017;(2):0067-0073. DOI. org/10.30567/RACI/20172/0067-0073. 